\title{
Nursing Staff Distress Associated With Neuropsychiatric Symptoms in Young-Onset Dementia and Late-Onset Dementia
}

Citation for published version (APA):

van Duinen-van den IJssel, J. C. L., Mulders, A. J. M. J., Smalbrugge, M., Zwijsen, S. A., Appelhof, B., Zuidema, S. U., de Vugt, M. E., Verhey, F. R. J., Bakker, C., \& Koopmans, R. T. C. M. (2018). Nursing Staff Distress Associated With Neuropsychiatric Symptoms in Young-Onset Dementia and Late-Onset Dementia. Journal of the American Medical Directors Association, 19(7), 627-632.

https://doi.org/10.1016/j.jamda.2017.10.004

Document status and date:

Published: 01/07/2018

DOI:

10.1016/j.jamda.2017.10.004

Document Version:

Publisher's PDF, also known as Version of record

Document license:

Taverne

Please check the document version of this publication:

- A submitted manuscript is the version of the article upon submission and before peer-review. There can be important differences between the submitted version and the official published version of record.

People interested in the research are advised to contact the author for the final version of the publication, or visit the DOI to the publisher's website.

- The final author version and the galley proof are versions of the publication after peer review.

- The final published version features the final layout of the paper including the volume, issue and page numbers.

Link to publication

\footnotetext{
General rights rights.

- You may freely distribute the URL identifying the publication in the public portal. please follow below link for the End User Agreement:

www.umlib.nl/taverne-license

Take down policy

If you believe that this document breaches copyright please contact us at:

repository@maastrichtuniversity.nl

providing details and we will investigate your claim.
}

Copyright and moral rights for the publications made accessible in the public portal are retained by the authors and/or other copyright owners and it is a condition of accessing publications that users recognise and abide by the legal requirements associated with these

- Users may download and print one copy of any publication from the public portal for the purpose of private study or research.

- You may not further distribute the material or use it for any profit-making activity or commercial gain

If the publication is distributed under the terms of Article $25 \mathrm{fa}$ of the Dutch Copyright Act, indicated by the "Taverne" license above, 
Original Study

\title{
Nursing Staff Distress Associated With Neuropsychiatric Symptoms in Young-Onset Dementia and Late-Onset Dementia
}

\author{
Jeannette C.L. van Duinen-van den IJssel MSc ${ }^{\text {a,b,*, Ans J.M.J. Mulders MD }}{ }^{\text {a,b,c }}$, \\ Martin Smalbrugge MD, PhD ${ }^{\mathrm{d}}$, Sandra A. Zwijsen MSc, PhD ${ }^{\mathrm{d}}$, Britt Appelhof MSc ${ }^{\mathrm{a}, \mathrm{b}, \mathrm{c}}$, \\ Sytse U. Zuidema MD, $\mathrm{PhD}^{\mathrm{e}}$, Marjolein E. de Vugt MSc, $\mathrm{PhD}^{\mathrm{f}}$, \\ Frans R.J. Verhey MD, PhD ${ }^{\mathrm{f}}$, Christian Bakker MSc, PhD ${ }^{\mathrm{a}, \mathrm{b}, \mathrm{g}}$, \\ Raymond T.C.M. Koopmans MD, PhD ${ }^{\text {a,b,h }}$
}

${ }^{a}$ Centre for Family Medicine, Geriatric Care and Public Health, Department of Primary and Community Care, Radboud University Nijmegen, Medical Centre, Nijmegen, the Netherlands

${ }^{\mathrm{b}}$ Radboudumc Alzheimer Centre, Nijmegen, The Netherlands

${ }^{\mathrm{c}}$ Archipel, Landrijt, Knowledge Centre for Specialized Care, Eindhoven, the Netherlands

${ }^{\mathrm{d}}$ Department of General Practice and Elderly Care Medicine, Amsterdam Public Health research institute, VU University Medical Center, Amsterdam, the Netherlands

e Department of General Practice and Elderly Care Medicine, University of Groningen, University Medical Centre Groningen, Groningen, the Netherlands

${ }^{\mathrm{f}}$ School for Mental Health and Neuroscience, Alzheimer Centre Limburg, Maastricht University Medical Centre, Maastricht, the Netherlands

${ }^{\mathrm{g}}$ Florence, Mariahoeve, Centre for Specialized Care in Young Onset Dementia, the Hague, the Netherlands

${ }^{\mathrm{h}}$ De Waalboog "Joachim en Anna," Centre for Specialized Geriatric Care, Nijmegen, the Netherlands

\section{Keywords:}

Nursing staff

distress

young-onset dementia

neuropsychiatric symptoms

long-term care

\section{A B S T R A C T}

Objective: The aims of this study were (1) to investigate the relationship between different neuropsychiatric symptoms (NPS) and the level of distress experienced by nurses caring for residents with youngonset dementia (YOD) and (2) to compare these findings with those for nurses caring for residents with late-onset dementia (LOD).

Design/Setting: This is a retrospective study conducted in Dutch long-term care facilities. Data were used from the Behavior and Evolution of Young-ONset Dementia studies (BEYOND) Parts I and II and the WAAL Behavior in Dementia-II (Waalbed-II) study.

Participants: A total of 382 nursing home residents with YOD and 261 nursing home residents with LOD were included.

Measurements: The Neuropsychiatric Inventory, nursing home version, was used to assess nursing staff distress and the frequency (F) and severity (S) of NPS. Multilevel logistic regression analysis was used to investigate the relationships between nursing staff distress related to NPS and YOD and LOD care units, the $\mathrm{F} \times \mathrm{S}$ score per symptom, gender, dementia subtype, and dementia severity.

Results: Nurses working in YOD care units rated sleep and nighttime behavior disorders, delusions, and agitation/aggression most often as highly distressing and euphoria most often as not distressing. Multivariate analyses indicated that the frequency and severity of NPS were significantly associated with staff distress in all symptoms, except for apathy. Comparison of the 2 groups of nurses demonstrated that the odds for distress related to sleep and nighttime behavior disorders were higher for nurses in YOD care units than for nurses in LOD units. For both the YOD and LOD nurses, irritability in male residents had higher impact than similar behavior in female residents.

Conclusion: This study provides important insight into distress related to individual NPS and the interaction with residents' characteristics. All NPS result in distress. The frequency and severity of the
This study was funded by the Netherlands Organization for Health Research and Development (ZonMW, no. 733050402), the Archipel Care Group in the Netherlands, the Florence Care Group in the Netherlands, the Dutch YOD Knowledge Center, and the Dutch Alzheimer Society.

The authors declare no conflicts of interest.

* Address correspondence to Jeannette C.L. van Duinen-van den IJssel, MSc, Department of Primary and Community Care, Centre for Family Medicine, Geriatric
Care and Public Health, Radboud University Medical Centre, Nijmegen, P.O. Box 9101, 6500 HB Nijmegen, The Netherlands.

E-mail address: Jeannette.vanduinen-vandenijssel@radboudumc.nl (J.C.L. van Duinen-van den IJssel). 
behavior is an important predictor. Sleep and nighttime behavior disorders are more likely to result in distress in YOD nurses than in LOD nurses. The amount of distress related to NPS emphasizes the urgent need for adequate management of NPS and the support of professional caregivers.

(c) 2017 AMDA - The Society for Post-Acute and Long-Term Care Medicine.

Caring for nursing home residents with dementia can be a demanding and challenging job. In particular, the presence of neuropsychiatric symptoms (NPS) is considered burdensome for nurses. ${ }^{1,2}$ For example, the burdens of NPS lead to sick days, lower quality of general health, increased workload, reduced work ability, and high burnout levels, likely leading to an increase in staff turnover. ${ }^{2-4}$ NPS are common in nursing home residents with dementia, even more so in people with young-onset dementia (YOD) in whom the first symptoms develop before the age of $65 .^{5}$ A large Dutch study on YOD nursing home residents concluded that nearly $90 \%$ of residents displayed NPS, with agitation/aggression and apathy being the most prevalent. ${ }^{6}$ Despite this high prevalence rate, research focusing on the impact of various NPS in YOD residents on nursing staff remains lacking.

Research on late-onset dementia (LOD) on this particular issue has primarily focused on the relation between aggressive behaviors and nursing staff stress. Aggression and the staff's perception of having little control over the behavior or the unpredictability of the behavior were most difficult to address. ${ }^{7}$ In addition, high caregiver burden was related to aggression that was perceived as threatening, verbal abuse, excessive demands, ill-natured denials, quarrelling, and screaming. ${ }^{8,9}$ Miyamoto et al. argued that in cases of aggressive behavior, nurses must manage this behavior while simultaneously protecting the other residents. ${ }^{8}$ Earlier studies found associations between aggressive and hostile behavior and the male gender. ${ }^{10,11}$ Furthermore, extreme agitation and aggressive behaviors appear more likely to occur in men below the age of $70 .^{12}$ For YOD, half of the residents are male, compared with a quarter in LOD. It is therefore likely that nurses caring for YOD residents must manage (extreme) aggressive behavior more often than nurses caring for residents with LOD.

Other NPS, such as disinhibition and irritability, are also distressing for nurses whereas apathy is most often considered the least distressing. ${ }^{2}$ Disinhibition is highly prevalent in YOD. ${ }^{6}$ This may be related to the prevalence of frontotemporal dementia (FTD), which is associated with disinhibition, wandering and stereotyping. ${ }^{13-15}$ NPS in people with YOD may have different effects because of increased physical fitness, such as walking speed, strength, and verbal abilities and because of the more emotional effect of the disease at a relatively young age. In YOD, there may be less acceptance of the decline and the shortened life expectancy by family or staff members. Furthermore, in YOD, there is more heterogeneity in diagnoses, ${ }^{6}$ and nurses may be confronted with more variation in NPS and dementia severity and be required to switch strategies and work routines, which may increase their workload, compared to nurses caring for LOD residents.

Over the past 10 years, increased attention to the specific needs of people with YOD led to the development of a national YOD care program by the Dutch YOD Knowledge Center (DKC). This resulted in the development of special care units (SCUs) with dedicated care for people with YOD. ${ }^{16}$ Therefore, in the Netherlands, there is a unique opportunity to investigate YOD nursing staff distress and compare their stress with the stress levels of the LOD nursing staff.

The aims of this study were (1) to investigate the relations between different NPS and the level of distress experienced by nurses caring for YOD nursing home residents and (2) to compare these findings with the distress experienced by nurses caring for people with LOD. This comparison may help design specific interventions to increase nursing staff knowledge and skills concerning NPS and thereby reduce distress and absenteeism and increase work ability in YOD SCUs.

\section{Methods}

\section{Study Design}

This study used baseline data from the Behavior and Evolution of Young-onset Dementia (Beyond) Parts $\mathrm{I}^{14}$ and $\mathrm{II}^{17}$ studies as well as baseline data from the WAAL Behavior in Dementia-II study. ${ }^{18}$ The Beyond-I study and the Waalbed-II study are descriptive studies investigating the characteristics and course of dementia and NPS in institutionalized people with YOD and people with LOD, respectively. ${ }^{14,18}$ The Beyond-II study is an intervention study focused on improving the management of NPS. ${ }^{17}$

The study protocols were all reviewed and approved by the Medical Ethics Committee region Arnhem/Nijmegen. All studies were conducted according to the principles of the Declaration of Helsinki (version November 2013, www.wma.net). In all 3 studies, informed consent was obtained from the first legal representative or legal guardian.

\section{Participants}

In the Beyond-I and Beyond-II studies, YOD nursing home residents were recruited from the YOD SCUs of nursing homes affiliated with the DKC. People were included in the study if they had an established dementia diagnosis with symptom onset before the age of 65 years and had been residing in the SCU for at least 1 month. Diagnoses of dementia subtype were made according to regular criteria and retrieved from the medical files. ${ }^{19-24}$ The exclusion criteria were lack of informed consent, dementia caused by human immunodeficiency virus (HIV), traumatic brain injury, Down syndrome, Korsakov syndrome, alcohol-related dementia, or Huntington disease. Some YOD SCUs participated in the Beyond-I as well as the Beyond-II study. When residents had the same gender, date of birth, and diagnosis, one of them was randomly excluded from either the Beyond-I or Beyond-II study to prevent duplication.

The LOD comparison group was selected from a historical cohort, the Waalbed-II study. Identical inclusion and exclusion criteria were applied as in the YOD group. Furthermore, residents from the LOD group were only included if they had an established symptom onset after the age of 65 or were older than 70 years at the time of institutionalization or at the time of inclusion in the Waalbed-II study. This regulation was enacted to reduce the risk of YOD residents being part of the LOD group.

\section{Data Collection Procedure}

All data were gathered by interviews with vocational nurses specifically assigned to the resident. Trained researchers and research assistants collected the data.

\section{Primary Outcome}

Distress was assessed using the occupational disruptiveness scale of the Dutch version of the Neuropsychiatric Inventory, Nursing Home version (NPI-NH). ${ }^{25-27}$ The NPI-NH assesses 10 behavioral symptoms: delusions, hallucinations, agitation/aggression, anxiety, depression, euphoria, apathy, disinhibition, irritability, aberrant motor behavior, and 2 neurovegetative symptoms: sleep and nighttime behavior disorders and appetite and eating changes. The NPI-NH has a high 
interrater reliability and is considered to be a valid measure for NPS. ${ }^{26}$ The occupational disruptiveness scale assesses the increase in work, effort, time or distress related to NPS on a 6-point scale, ranging from not disruptive at all to very severely to extremely disruptive $(0-5){ }^{25,27}$ The total distress score can be calculated by totaling the 12 distress scores, range 0-60.

\section{Independent Variables}

The NPI-NH was also used to assess the frequency $(F)$ and severity $(S)$ of NPS on a 4-point (1-4) and 3-point (1-3) Likert-type scale, respectively. ${ }^{25-27}$ A total NPI-NH score can be calculated by multiplying $\mathrm{F}$ by $\mathrm{S}$; the $\mathrm{F} \times \mathrm{S}$ scores range from 0 (symptom was absent) to 12 .

The severity of dementia was assessed with the Global Deterioration Scale (GDS) ${ }^{28}$ The GDS is a validated 7-point scale that describes 7 different stages of dementia ranging from no cognitive decline (1) to very severe cognitive decline (7). Stages 4 and higher are considered to represent subsequent dementia stages. The GDS is a widely used instrument and has been validated against behavioral, neuroanatomic, and neuropsychological measures. ${ }^{28}$ The residents' year of first symptoms of dementia, age, gender, and dementia subtype were obtained from the medical file.

\section{Data Analysis}

Analyses were conducted using the Statistical Package for Social Sciences, version 22. ${ }^{29}$ Before analysis, data were checked for missing values, outliers, duplicates, linearity, and normality. Residents with missing scores on the NPI-NH or GDS were excluded from the analysis. Only residents with an F x S score $\geq 1$ on at least one of the NPI items were included in the analysis because only then could a distress score be obtained. Demographic variables of the YOD and the LOD groups were described using means or frequencies. The distribution of distress ratings among nurses caring for YOD and LOD residents were categorized as low (score $0-1$ ), medium (score 2-3), and high distress (score 4-5). ${ }^{30}$ Mean total distress scores in the YOD and LOD groups were calculated and compared with the independent samples $t$ test.

Because the distress scores were not normally distributed, a multilevel logistic model was used to compare distress related to NPS in nurses caring for YOD and nurses caring for LOD residents. For this analysis the dependent variable distress was dichotomized into no distress (0-1) and distress (2-5). Odds ratios were calculated for group (YOD/LOD), $\mathrm{F} \times \mathrm{S}$ score, gender, dementia severity (GDS: 2-5, 6-7), and dementia subtype (Alzheimer's disease, Vascular/Mixed and Other [comprising of FTD, Lewy body dementia, Parkinson dementia, neurosyphilis, and dementia not otherwise specified]). The multilevel model corrected for clustering of residents within nursing homes. Additional subgroup analyses were performed to explore whether the effect of the group (YOD or LOD) was different by dementia subtype, gender, $\mathrm{F} \times \mathrm{S}$ scores and dementia severity. Therefore, the interaction terms group*gender, group*dementia subtype, group* $\mathrm{F} \times \mathrm{S}$ score and group*dementia severity were added to the multilevel logistic model and manually analyzed using backward selection. Only significant interactions were included in the final analysis. All analyses were 2 -tailed, and a $P$ value of $<.05$ was considered statistically significant.

\section{Results}

\section{Sociodemographic Characteristics}

In this study, a total of 382 residents with YOD and 261 residents with LOD were included. Approximately half of the YOD residents were male compared with nearly one-quarter of the LOD residents
Table 1

Demographic and Clinical Characteristics of YOD and LOD Residents

\begin{tabular}{lll}
\hline & YOD $(\mathrm{n}=382)$ & LOD $(\mathrm{n}=261)$ \\
\hline Resident age at inclusion & & \\
Mean (SD) & $62.7(6.9)$ & $84.9(5.9)$ \\
Range & $39.3-78.2$ & $70.4-102.2$ \\
Gender, male, n (\%) & $193(50.5)$ & $59(22.6)$ \\
Dementia subtype, $\mathrm{n}(\%)$ & & \\
Alzheimer's disease & 41.9 & 43.7 \\
Vascular dementia and mixed dementia & 16.5 & 16.9 \\
Frontotemporal dementia & 24.6 & 3.1 \\
Other & 17 & 36.4 \\
Dementia severity (GDS), n (\%) & & \\
Mild (2-4) & 16 & 5.7 \\
Moderate (5) & 21.5 & 23.4 \\
Severe (6) & 35.9 & 42.9 \\
Very severe (7) & 26.7 & 28 \\
NPI-NH F $\times$ S score, mean (SD) & & \\
Delusions & $6.42(4.0)$ & $5.10(3.2)$ \\
Hallucinations & $5.34(3.5)$ & $3.49(2.1)$ \\
Agitation/aggression & $6.44(3.4)$ & $5.23(3.4)$ \\
Depression & $5.32(3.4)$ & $4.45(2.8)$ \\
Anxiety & $6.77(3.5)$ & $5.47(3.0)$ \\
Euphoria & $5.57(3.2)$ & $4.31(2.0)$ \\
Apathy & $8.28(3.4)$ & $7.18(3.6)$ \\
Disinhibition & $6.82(3.8)$ & $6.06(3.3)$ \\
Irritability & $6.27(3.2)$ & $5.56(3.3)$ \\
Aberrant motor behavior & $8.77(3.5)$ & $7.55(3.2)$ \\
Sleep and nighttime behavior disorders & $6.19(3.3)$ & $4.96(3.1)$ \\
Appetite and eating changes & $8.05(3.5)$ & $6.65(2.8)$ \\
\hline & &
\end{tabular}

(Table 1 ). The mean age of the YOD group was 62.7 years [standard deviation (SD) 6.9] and 84.9 years (SD 5.9) in the LOD group. The prevalence of Alzheimer's dementia, vascular dementia, and mixed dementia was nearly equal in both groups. However, FTD was more prevalent in YOD residents (24.6\% vs 3.1\%). Most residents in both groups had (very) severe dementia (GDS score 6 or higher). However, in the YOD group, more residents had mild dementia (16\%) compared with the LOD group (5.7\%). In YOD and LOD, the mean $\mathrm{F} \times \mathrm{S}(\mathrm{SD})$ score was highest for aberrant motor behavior [8.8 (3.5) and 7.55 (3.2)], followed by apathy at 8.28 (3.4) and 7.18 (3.6) and appetite and eating changes at 8.05 (3.5) and 6.65 (2.8). In YOD, the mean $\mathrm{F} \times \mathrm{S}$ score was lowest for depression at 5.32 (3.4), whereas in LOD, this was lowest for hallucinations at $3.49(2.1)$.

\section{NPS and Distress in YOD and LOD Nursing Staff}

In both YOD and LOD residents, agitation/aggression, apathy, and irritability were the most common behavioral symptoms (Table 2). All separate NPS caused medium or high distress in a larger majority of nurses caring for YOD residents (Table 2). Approximately $45 \%$ of the nurses rated sleep and nighttime behavior disorders, agitation/ aggression, and delusions as highly distressing, and a nearly equal percentage rated these behaviors to be medium distressing. Euphoria was most often rated as not distressing. However, more than half of the nurses experienced some level of distress because of euphoria. Furthermore, although distress related to hallucinations was often rated as absent or minimal, a similar percentage rated hallucinations as medium distressing. All other symptoms were most often rated as medium distressing by nurses in YOD care units.

As in YOD, all symptoms cause distress in LOD. However, for all symptoms, the absolute rates of high distress are lower for LOD, and the rates of no/low distress are higher for LOD. When distress was experienced, it was for all symptoms most often rated as medium distressing. More than half of the nurses caring for LOD residents rated anxiety, irritability, and appetite and eating changes to be medium distressing. More than half of the nurses experienced no/low distress related to hallucinations, euphoria, and apathy. Comparison of the 
Table 2

Prevalence of Individual NPS in YOD and LOD Residents and Corresponding Distribution (Percentages) of Nursing Staff Distress

\begin{tabular}{|c|c|c|c|c|}
\hline Symptoms & $\mathrm{n}(\%)$ & $\begin{array}{l}\text { No/Low } \\
\text { Distress, \% }\end{array}$ & $\begin{array}{l}\text { Medium } \\
\text { Distress, \% }\end{array}$ & $\begin{array}{l}\text { High } \\
\text { Distress, \% }\end{array}$ \\
\hline \multicolumn{5}{|l|}{ Delusions } \\
\hline YOD & $59(15.4)$ & 16.9 & 39 & 44.1 \\
\hline LOD & $49(18.8)$ & 30.6 & 38.8 & 30.6 \\
\hline \multicolumn{5}{|c|}{ Hallucinations } \\
\hline YOD & $56(14.7)$ & 41.1 & 42.9 & 16.1 \\
\hline LOD & 39 (14.9) & 53.8 & 33.3 & 12.8 \\
\hline \multicolumn{5}{|c|}{ Agitation and aggression } \\
\hline YOD & $201(52.6)$ & 10.9 & 44.8 & 44.3 \\
\hline LOD & $111(42.5)$ & 25.2 & 46.8 & 27.9 \\
\hline \multicolumn{5}{|l|}{ Depression } \\
\hline YOD & $117(30.7)$ & 21.4 & 49.6 & 29.1 \\
\hline LOD & $74(28.4)$ & 36.5 & 47.3 & 16.2 \\
\hline \multicolumn{5}{|l|}{ Anxiety } \\
\hline YOD & $84(22)$ & 15.5 & 51.2 & 33.3 \\
\hline LOD & $70(26.8)$ & 20 & 58.6 & 21.4 \\
\hline \multicolumn{5}{|l|}{ Euphoria } \\
\hline YOD & $61(16)$ & 45.9 & 41 & 13.1 \\
\hline LOD & $16(6.1)$ & 62.5 & 31.3 & 6.3 \\
\hline \multicolumn{5}{|l|}{ Apathy } \\
\hline YOD & $200(52.5)$ & 36 & 49 & 15 \\
\hline LOD & $92(35.2)$ & 51.2 & 41.3 & 7.6 \\
\hline \multicolumn{5}{|c|}{ Disinhibition } \\
\hline YOD & $136(35.7)$ & 22.1 & 38.2 & 39.7 \\
\hline LOD & $49(18.8)$ & 30.6 & 44.9 & 24.5 \\
\hline \multicolumn{5}{|l|}{ Irritability } \\
\hline YOD & $193(50.6)$ & 14 & 54.9 & 31.1 \\
\hline LOD & $105(40.2)$ & 22.9 & 51.4 & 25.7 \\
\hline \multicolumn{5}{|c|}{ Aberrant motor behavior } \\
\hline YOD & $161(42.3)$ & 29.2 & 40.4 & 30.4 \\
\hline LOD & $73(28)$ & 42.5 & 37 & 20.5 \\
\hline \multicolumn{5}{|c|}{$\begin{array}{l}\text { Sleep and nighttime behavior } \\
\text { disorders }\end{array}$} \\
\hline YOD & $74(19.4)$ & 10.8 & 43.2 & 45.9 \\
\hline LOD & $52(19.9)$ & 36.5 & 36.5 & 26.9 \\
\hline \multicolumn{5}{|c|}{ Appetite and eating changes } \\
\hline YOD & 76 (19.9) & 13.2 & 52.6 & 34.2 \\
\hline LOD & $55(21.1)$ & 25.5 & 61.8 & 12.7 \\
\hline
\end{tabular}

mean total distress between YOD and LOD nursing staff indicated that nurses caring for YOD residents experienced significantly $[t(608.236)=5.157, P=.0001]$ more distress $(\mathrm{M}=10.0, \mathrm{SD}=8.6)$ than nurses caring for LOD residents $(\mathrm{M}=6.8, \mathrm{SD}=7.4)$.
The multilevel logistic regression analyses (Table 3) indicated that the odds of experiencing distress related to sleep and nighttime behavior disorders was significantly higher for the YOD nursing staff than for the LOD nursing staff [sleep and nighttime behavior disorders odds ratio (OR): $3.93,95 \%$ confidence interval (CI): 1.37-11.3; $P=.012$ ]. For all other symptoms, no significant differences were found between YOD and LOD nursing staff.

Our analysis also indicated that after controlling for group (YOD vs LOD), gender, dementia subtype, and dementia severity, for every 1point increase in the $\mathrm{F} \times \mathrm{S}$ score, nurses were more likely to experience distress. This was found for all symptoms with the exception of apathy (Table 3). The OR was lowest for aberrant motor behavior (OR: 1.30, 95\% CI: 1.18-1.43; $P=.0001$ ) and highest for irritability (OR: 1.59 , 95\% CI: 1.34-1.87, $P=.0001$ ).

Additionally, in this model we found an association between gender and distress related to irritability (OR: 2.57, 95\% CI: 1.15 - 5.76; $P=.022$ ). In both groups of nurses, the odds of experiencing distress related to irritability increased when male residents were compared with female residents. No significant effects were identified between dementia severity and distress and dementia subtype and distress.

Additional subgroup analysis (not shown) revealed no interaction effects between group and gender, dementia subtype, dementia severity, and $\mathrm{F} \times \mathrm{S}$ score.

\section{Discussion}

This study is the first to address nursing staff distress related to NPS in YOD and to compare these figures with the LOD nursing staff. We found that all NPS affect nurses caring for YOD residents as well as nurses caring for LOD residents. Most YOD nurses experienced medium or high levels of distress for all symptoms, which indicates that according to the NPI-NH, NPS are quite disruptive and upsetting to staff and a major time infringement. ${ }^{25,27}$ LOD nurses also experienced distress related to all different symptoms, although they less often appeared to experience high levels of distress. In both YOD and LOD residents, sleep and nighttime behavior disorders, agitation/ aggression, and delusions were the most distressing NPS. In YOD, these symptoms were most often rated as highly distressing by nurses, whereas in LOD these symptoms were most often rated as medium distressing. Consistent with earlier studies, we found that agitation/aggression and sleep and nighttime behavior disorders in

Table 3

ORs and 95\% CIs of the Relationship Between the Independent Variables and Distress of the Individual Neuropsychiatric Symptoms

\begin{tabular}{|c|c|c|c|c|c|c|c|}
\hline & Delusions & Hallucinations & \multirow{2}{*}{\multicolumn{2}{|c|}{$\frac{\text { Agitation/Aggression }}{\mathrm{OR}(95 \% \mathrm{CI})}$}} & Depression & Anxiety & Euphoria \\
\hline & OR (95\% CI) & OR (95\% CI) & & & OR (95\% CI) & OR $(95 \% \mathrm{CI})$ & OR $(95 \% \mathrm{CI})$ \\
\hline Group (ref: LOD) & $2.30(0.50-10.69)$ & $0.92(0.24-3.54)$ & \multicolumn{2}{|l|}{$1.49(0.72-3.08)$} & $2.38(0.95-5.98)$ & $1.03(0.38-2.75)$ & $1.06(0.20-5.76)$ \\
\hline $\mathrm{F} \times \mathrm{S}$ score & $1.47(1.16-1.86)$ & $1.40(1.15-1.7)$ & \multicolumn{2}{|l|}{$1.55(1.34-1.80)$} & $1.45(1.23-1.72)$ & $1.38(1.15-1.64)$ & $1.56(1.19-2.03)$ \\
\hline Gender (ref: female) & $0.74(0.22-2.46)$ & $1.43(0.51-3.99)$ & \multicolumn{2}{|l|}{$1.53(0.73-3.23)$} & $0.49(0.21-1.11)$ & $1.06(0.34-3.24)$ & $1.23(0.33-4.60)$ \\
\hline Dementia severity (ref: 6-7) & $0.52(0.14-1.97)$ & $0.72(0.15-3.46)$ & \multicolumn{2}{|l|}{$1.41(0.67-2.96)$} & $0.63(0.30-1.36)$ & $1.61(0.51-5.11)$ & $0.64(0.17-2.39)$ \\
\hline \multicolumn{8}{|l|}{ Dementia subtype (ref: AD) } \\
\hline Vasc./mixed & $1.65(0.19-14.56)$ & $0.89(0.21-3.79)$ & \multicolumn{2}{|l|}{$1.23(0.47-3.23)$} & $1.47(0.55-3.91)$ & $0.96(0.27-3.37)$ & $0.24(0.04-1.58)$ \\
\hline \multirow[t]{3}{*}{ Other } & $0.64(0.18-2.25)$ & $0.61(0.18-2.05)$ & $0.63(0.29-1.34)$ & & $1.11(0.47-2.65)$ & $0.54(0.19-1.51)$ & $2.03(0.52-7.98)$ \\
\hline & Apathy & Disinhibition & Irritability & \multicolumn{2}{|c|}{ Aberrant Motor Behavior } & $\begin{array}{l}\text { Sleep and Nighttime } \\
\text { Behavior Disorders }\end{array}$ & $\begin{array}{l}\text { Appetite and } \\
\text { Eating Changes }\end{array}$ \\
\hline & OR (95\% CI) & OR $(95 \% \mathrm{CI})$ & OR (95\% CI) & \multicolumn{2}{|c|}{ OR (95\% CI) } & OR $(95 \% \mathrm{CI})$ & OR $(95 \% \mathrm{CI})$ \\
\hline Group (ref: LOD) & $1.34(0.58-3.10)$ & $1.37(0.57-3.29)$ & $0.98(0.38-2.49)$ & \multicolumn{2}{|c|}{$1.49(0.61-3.64)$} & $3.93(1.37-11.3)$ & $1.81(0.58-5.60)$ \\
\hline $\mathrm{F} \times \mathrm{S}$ score & $1.02(0.94-1.11)$ & $1.55(1.32-1.82)$ & $1.59(1.34-1.87)$ & \multicolumn{2}{|c|}{$1.30(1.18-1.43)$} & $1.56(1.24-1.95)$ & $1.36(1.13-1.63)$ \\
\hline Gender (ref: female) & $1.06(0.62-1.82)$ & $1.02(0.46-2.29)$ & $2.57(1.15-5.76)$ & \multicolumn{2}{|c|}{$0.82(0.42-1.60)$} & $0.95(0.33-2.71)$ & $1.92(0.56-6.64)$ \\
\hline Dementia severity (ref: 6-7) & $1.07(0.55-2.12)$ & $1.14(0.49-2.62)$ & $1.36(0.62-3.01)$ & \multicolumn{2}{|c|}{$0.61(0.29-1.30)$} & $0.96(0.30-3.04)$ & $0.67(0.24-1.82)$ \\
\hline \multicolumn{8}{|l|}{ Dementia subtype (ref: AD) } \\
\hline Vasc./Mixed & $0.67(0.31-1.45)$ & $0.75(0.24-2.33)$ & $0.50(0.19-1.33)$ & \multicolumn{2}{|c|}{$0.86(0.31-2.39)$} & $1.86(0.44-7.83)$ & $0.55(0.12-2.48)$ \\
\hline Other & $1.20(0.68-2.11)$ & $1.22(0.49-3.01)$ & $0.60(0.25-1.42)$ & \multicolumn{2}{|c|}{$1.41(0.70-2.85)$} & $1.49(0.44-7.83)$ & $0.41(0.12-1.34)$ \\
\hline
\end{tabular}

AD, Alzheimer's disease; Vasc., vascular dementia.

Bold indicates significant result with $P<.05$. 
particular appeared to cause distress in nurses. ${ }^{2,8,9}$ Sleep and nighttime behavior disorders and delusions, although in our sample relatively low in prevalence rate, caused high levels of distress in YOD nurses. Matsumoto et al. concluded that even if the frequency and severity of symptoms were low, they nevertheless caused substantial distress. ${ }^{31}$

Consistent with earlier studies, we found that the likelihood of experiencing distress increased when the frequency and/or severity of NPS increased. ${ }^{2}$ We did not identify a relation between the $\mathrm{F} \times \mathrm{S}$ score for apathy and the corresponding distress score, despite the high prevalence of apathy and high mean apathy scores in both groups. Zwijsen et al. argued that apathy most likely does not distress nurses. ${ }^{2}$ Indeed, it is likely that nurses are not required to intervene or change their work routines because of apathy in residents. However, it is possible that apathy influences the quality of life of the resident. A recent study related apathy in YOD to a lower quality of life. ${ }^{32}$ Other researchers found that the relationship between apathy in people with dementia and quality of life depended on the level of cognitive functioning and only was associated with quality of life in nursing home residents with better cognitive functioning. ${ }^{33,34}$ This suggests that health care professionals should evaluate for each case individually whether or not a person with dementia experiences a lower quality of life as a result of apathy.

We found that distress related to sleep and nighttime behavior disorders was specifically dependent on YOD or LOD, after correction for other factors. The NPI-NH subscale Sleep and Nighttime Behavior Disorders assesses behaviors such as wandering, pacing, waking up too early, and going to other rooms. ${ }^{25,27,30}$ From a clinical perspective, people with YOD are more physically fit and are more often able to get out of bed compared with people with LOD. Therefore, nighttime behavior in YOD may be more disturbing to the social environment. In most nursing homes, only a few nurses work during the evening and night; consequently, managing this behavior requires changing their work routine and can be time-consuming and therefore particularly burdensome for nurses. Multivariate analysis in our study did not show differences in nursing staff distress related to agitation/aggression between nurses caring for either YOD or LOD residents. This is surprising, given the fact that health care professionals in clinical practice report that being hit by a young person is perceived differently than being hit by an older person, probably because younger people are more vital and strong. Maybe not the younger age per se but the physical characteristics of the younger person might be more important such as strength, vitality, mobility, and body size of the person with dementia. Future research should take this into account.

The likelihood of experiencing irritability as distressing was higher when presenting in males than in females. This is partially consistent with a study in which residents' irritability was found to be distressing for nurses, ${ }^{2}$ and a study associating irritability in community-dwelling people with dementia with informal caregiver distress. ${ }^{31}$ However, those studies did not discriminate by gender. One study on LOD found that males became annoyed more easily than females although that study did not investigate nursing staff burden. ${ }^{10}$ Future research should further investigate this phenomenon.

Our study is unique in that to our knowledge, we included the largest group of institutionalized people with YOD and their nurses and directly compared our data with a similar LOD study sample. However, there are also some limitations that must be discussed. First, we dichotomized distress scores for statistical reasons, which results in a loss of information. Consequently, we were not able to compare nurses who rated NPS as non-, medium, or highly distressing. In addition, some NPS were displayed only by a few YOD and/or LOD residents; thus, we had to collapse groups to increase power, reducing dementia severity to only 2 groups. Furthermore, we asked the vocational nurses specifically assigned to the residents to rate the behavior and the effects of the behavior. It is possible that the degree to which a nurse was distressed by NPS influenced the ratings of the frequency and severity of NPS. Finally, we combined 3 datasets, of which the Beyond-I and Waalbed-II studies were of the same cohort and the Beyond-II study was of a newer cohort. ${ }^{14,17,18}$ This may have caused an unwanted cohort effect because in recent years, nursing homes have paid more attention to the recognition and treatment of NPS. However, because we chose multilevel analysis in which we corrected for the nursing home, we partially corrected for this.

Future research should focus on using a combination of quantitative and qualitative measurement instruments because some symptoms can be emotionally difficult to address whereas other symptoms lead to an increased workload. For deeper understanding of the emotional impact of NPS on nurses, structured interviews could be used to investigate the experiences of nurses concerning the resident's behavior. This should include the actual moment residents exhibit a certain behavior but also the period thereafter, because some behaviors can have a lasting impact. Furthermore, characteristics of the nurses should be considered. Hazelhof et al. demonstrated that distress related to NPS was also influenced by the characteristics of the nurses. ${ }^{1}$ Knowledge regarding the content of distress and the influence of nurses' characteristics renders it possible to develop specific interventions aimed at decreasing distress.

\section{Conclusion}

This explorative study illustrates the effect on nursing staff of NPS in residents with YOD. Nurses experienced distress related to NPS, and a fair number of nurses experienced high levels of distress related to agitation/aggression, delusions, and sleep and nighttime behavior disorders. Nurses on YOD care units must be better supported in addressing residents' sleep and nighttime behaviors. In addition, interventions can be developed to focus on increasing the night rest of the residents. Addressing NPS is a major portion of nurses' daily jobs, and the amount of distress related to NPS emphasizes the urgent need for adequate management of NPS and support for professional caregivers. Moreover, psychosocial interventions as treatment of NPS are mostly conducted by nurses. Distress in nurses can affect the quality of care and maybe even increase NPS in the resident. Therefore, nursing staff distress should be part of the treatment of NPS. Finally, some symptoms cause little distress in nurses, such as apathy and euphoria. However, because they can affect the quality of life of the resident, it is important to be aware of the signs of these NPS.

\section{References}

1. Hazelhof TJ, Schoonhoven L, van Gaal BG, et al. Nursing staff stress from challenging behaviour of residents with dementia: A concept analysis. Int Nurs Rev 2016;63:507-516.

2. Zwijsen SA, Kabboord A, Eefsting JA, et al. Nurses in distress? An explorative study into the relation between distress and individual neuropsychiatric symptoms of people with dementia in nursing homes. Int J Geriatr Psychiatry 2014;29:384-391.

3. Kennedy BR. Stress and burnout of nursing staff working with geriatric clients in long-term care. J Nurs Scholarsh 2005;37:381-382.

4. Schmidt SG, Dichter MN, Palm R, Hasselhorn HM. Distress experienced by nurses in response to the challenging behaviour of residents-Evidence from German nursing homes. J Clin Nurs 2012;21:3134-3142.

5. World Health Organization. Mental health: Dementia report, 2012, http://www. who.int/mental_health/publications/dementia_report_2012/en/. Accessed March 3, 2017.

6. Mulders AJ, Fick IW, Bor H, et al. Prevalence and correlates of neuropsychiatric symptoms in nursing home patients with young-onset dementia: The BEYOnD study. J Am Med Dir Assoc 2016;17:495-500.

7. Brodaty H, Draper B, Low LF. Nursing home staff attitudes towards residents with dementia: Strain and satisfaction with work. J Adv Nurs 2003;44: 583-590.

8. Miyamoto Y, Tachimori H, Ito H. Formal caregiver burden in dementia: Impact of behavioral and psychological symptoms of dementia and activities of daily living. Geriatr Nurs 2010;31:246-253. 
9. Rodney V. Nurse stress associated with aggression in people with dementia: Its relationship to hardiness, cognitive appraisal and coping. J Adv Nurs 2000;31: $172-180$.

10. Lovheim H, Sandman PO, Karlsson S, Gustafson Y. Sex differences in the prevalence of behavioral and psychological symptoms of dementia. Int Psychogeriatr 2009;21:469-475.

11. Zuidema SU, de Jonghe JF, Verhey FR, Koopmans RT. Predictors of neuropsychiatric symptoms in nursing home patients: Influence of gender and dementia severity. Int J Geriatr Psychiatry 2009;24:1079-1086.

12. Brodaty H, Draper BM, Low LF. Behavioural and psychological symptoms of dementia: A seven-tiered model of service delivery. Med J Aust 2003;178: $231-234$

13. Bathgate D, Snowden JS, Varma A, et al. Behaviour in frontotemporal dementia, Alzheimer's disease and vascular dementia. Acta Neurol Scand 2001;103: 367-378.

14. Mulders AJ, Zuidema SU, Verhey FR, Koopmans RT. Characteristics of institutionalized young onset dementia patients - the BEYOnD study. Int Psychogeriatr 2014;26:1973-1981.

15. Rossor MN, Fox NC, Mummery CJ, et al. The diagnosis of young-onset dementia. Lancet Neurol 2010;9:793-806.

16. Carter JE, Oyebode JR, Koopmans RT. Young-onset dementia and the need for specialist care: A national and international perspective. Aging Ment Health; 2017:1-6.

17. van Duinen-van den IJssel JCL, Appelhof B, Zwijsen SA, et al. Behavior and Evolution of Young ONset Dementia part 2 (BEYOND-II) study: An intervention study aimed at improvement in the management of neuropsychiatric symptoms in institutionalized people with young onset dementia. Int Psychogeriatr; 2017:1-10.

18. Wetzels RB, Zuidema SU, de Jonghe JF, et al. Course of neuropsychiatric symptoms in residents with dementia in nursing homes over 2-year period. Am J Geriatr Psychiatry 2010;18:1054-1065.

19. American Psychiatric Association. Diagnostic and Statistical Manual of Mental Disorders-Text Revision: DSM-IV-TR. Washington, DC: American Psychiatric Association; 2000

20. Gorno-Tempini ML, Hillis AE, Weintraub S, et al. Classification of primary progressive aphasia and its variants. Neurology 2011;76:1006-1014.

21. McKeith IG. Consensus guidelines for the clinical and pathologic diagnosis of dementia with Lewy bodies (DLB): Report of the Consortium on DLB International Workshop. J Alzheimers Dis 2006;9:417-423.
22. McKhann GM, Knopman DS, Chertkow H, et al. The diagnosis of dementia due to Alzheimer's disease: Recommendations from the National Institute on Aging-Alzheimer's Association workgroups on diagnostic guidelines for Alzheimer's disease. Alzheimers Dement 2011;7:263-269.

23. Rascovsky K, Hodges JR, Knopman D, et al. Sensitivity of revised diagnostic criteria for the behavioural variant of frontotemporal dementia. Brain 2011; 134:2456-2477.

24. Roman GC, Tatemichi TK, Erkinjuntti T, et al. Vascular dementia: Diagnostic criteria for research studies. Report of the NINDS-AIREN International Workshop. Neurology 1993;43:250-260.

25. Cummings JL, Mega M, Gray K, et al. The neuropsychiatric inventory: Comprehensive assessment of psychpathology in dementia. Neurology 1994; 44:2308-2314.

26. Kat MG, de Jonghe JF, Aalten P, et al [Neuropsychiatric symptoms of dementia: Psychometric aspects of the Dutch Neuropsychiatric Inventory (NPI)]. Tijdschr Gerontol Geriatr 2002;33:150-155.

27. Wood S, Cummings JL, Hsu MA et al. The use of the neuropsychiatric inventory in nursing home residents. Characterization and measurement. Am J Geriatr Psychiatry 2000;8:75-83.

28. Reisberg B, Ferris SH, de Leon MJ, Crook T. The Global Deterioration Scale for assessment of primary degenerative dementia. Am J Psychiatry 1982;139: 1136-1139.

29. IBM SPSS Statistics for Windows, version 22.0 [computer program]. Armonk, NY: IBM Corp; 2013.

30. Kaufer DI, Cummings JL, Christine D, et al. Assessing the impact of neuropsychiatric symptoms in Alzheimer's disease: The Neuropsychiatric Inventory Caregiver Distress Scale. J Am Geriatr Soc 1998:46:210-215.

31. Matsumoto N, Ikeda M, Fukuhara R, et al. Caregiver burden associated with behavioral and psychological symptoms of dementia in elderly people in the local community. Dement Geriatr Cogn Disord 2007;23:219-224.

32. Appelhof B, Bakker C, Van Duinen-van den IJssel JCL, et al. The determinants of quality of life of nursing home residents with young-onset dementia and the differences between dementia subtypes. Dement Geriatr Cogn Disord 2017;43: $320-329$.

33. Gerritsen DL, Jongenelis K, Steverink N, et al. Down and drowsy? Do apathetic nursing home residents experience low quality of life? Aging Ment Health 2005:9:135-141.

34. Yeager CA, Hyer L. Apathy in dementia: Relations with depression, functional competence, and quality of life. Psychol Rep 2008;102:718-722. 